\title{
Investigation of Various Types of Inverse Micelles in Nonpolar Liquids Using Transient Current Measurements
}

\author{
Masoumeh Karvar, ${ }^{, \dagger}$ Filip Strubbe, ${ }^{\dagger, \grave{\ddagger}}$ Filip Beunis, ${ }^{\dagger, \dagger}$ Roger Kemp, ${ }^{\S}$ Nathan Smith, ${ }^{\S}$ Mark Goulding, ${ }^{\S}$ \\ and Kristiaan Neyts ${ }^{\dagger, t}$ \\ ${ }^{\dagger}$ Department of Electronics and Information Systems and Center for Nano- and Biophotonics, Ghent University, B-9000 Ghent, \\ Belgium \\ ${ }^{\S}$ Merck Chemicals Ltd, University Parkway, Chilworth, Southampton SO16 7QD, United Kingdom
}

ABSTRACT: Transient current measurements are used to characterize a wide variety of charge carriers in nonpolar liquids. The transient current method allows us to obtain both the concentration and mobility of charge carriers and therefore also the hydrodynamic radius using Stokes' law. In this article, five different surfactants in dodecane are investigated: OLOA11K, Solsperse13940, Span80, Span85, and AOT. We show that different types of currents are observed depending on the size of the inverse micelles. For large inverse micelles such as for OLOA11K, Solsperse13940, and Span80,
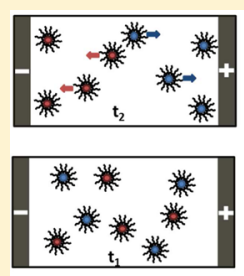

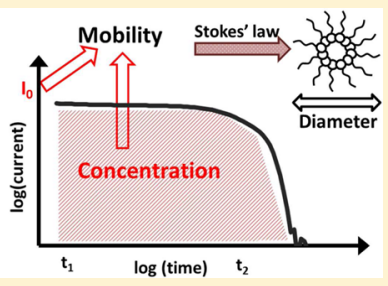
the measurement of the transient current is straightforward because of the low steady-state current level. However, for small inverse micelles such as AOT and Span85, the current from the generation of charges is much larger such that high voltages, a small distance between the electrodes, and dielectric coatings on the electrodes are required to measure the signal related to the initially present charged inverse micelles. The estimated hydrodynamic radii of AOT and Span85, the two smallest inverse micelles, are in good agreement with the values reported in the literature. The comparison of the transient currents with simulations indicates that the dynamics of the charge transport are well-understood.

\section{INTRODUCTION}

The increased interest in surfactants in nonpolar liquids in the past decade has led to the need for adequate characterization techniques. Surfactants are used as detergents, wetting agents, emulsifiers, foaming agents, and dispersants. In the case of inks for electrophoretic ink displays, ${ }^{1-8}{ }^{\text {inkjet printing, }}{ }^{9}$ and liquid toner printing, ${ }^{10}$ surfactants are used to sterically stabilize and charge pigment particles. The electrical behavior of such nonpolar colloids is strongly influenced by the presence of charged species in nonpolar media in the form of charged inverse micelles. ${ }^{11}$

Inverse micelles consist of aggregated surfactant molecules, with the hydrophilic heads pointing inward and the hydrophobic tails sticking outward into the nonpolar medium. In thermal equilibrium, a small fraction of these inverse micelles carry an electrical charge. ${ }^{11,12}$ This results in a relatively high conductivity for surfactant dispersions in nonpolar media, ${ }^{13,14}$ which affects both the transport of colloidal particles and the energy consumption of electronic ink devices.

Inverse micelles in nonpolar solvents can be investigated and characterized with different methods. Static (SLS) and dynamic (DLS) light scattering and viscosity measurements are often used to study the size and shape of inverse micelles. ${ }^{15-21}$ For instance, with DLS measurement, Guo et al. determined the hydrodynamic radius of Span 58 in hexane to be $1.4 \mathrm{~nm},{ }^{22}$ and Eicke et al. reported a value of $1.6 \mathrm{~nm}$ for the hydrodynamic radius of AOT. ${ }^{23}$ The small-angle neutron scattering (SANS) technique is a method used to study inverse micelles and to estimate their size, shape, aggregation number, and internal structure. Kotlarchyk et al. used this method to characterize AOT inverse micelles in $n$-decane. ${ }^{24}$ They found that AOT inverse micelles are approximately spherical, with an aggregation number of about 22 monomers per micelle and a hydrodynamic radius of $1.6 \mathrm{~nm}$.

The previously mentioned methods measure charged and uncharged inverse micelles simultaneously. Because only a small portion of the inverse micelles carry a charge, the results from these methods mainly represent the uncharged inverse micelles. Conductivity measurements are one of the more popular methods for studying the nature and the concentration of charged inverse micelles of surfactants in nonpolar liquids. ${ }^{9,25,26}$ The dependency of the conductivity on the surfactant concentration can be used to study the charging mechanism (disproportionation or dissociation model). ${ }^{27-29}$ Assuming that all of the inverse micelles have the same size and a valency of $\pm 1,{ }^{12,30}$ the conductivity is proportional to the products of the elementary charge, the concentration, and the mobility of the charge carriers. From the conductivity measurement we can find the product of the concentration and the mobility, but an additional measurement is needed to determine both quantities separately. ${ }^{27,28}$

In the following we focus on the analysis of transient current measurements that can be used to determine the conductivity $\sigma$, the concentration $n_{ \pm}$, and the mobility $\mu_{ \pm}$of charged inverse

Received: June 11, 2014

Revised: August 25, 2014

Published: September 25, 2014 
micelles. This technique has proven to be successful in characterizing charged inverse micelles with low steady-state currents (low bulk and surface generation currents). ${ }^{11,29}$ In these experiments, a voltage is applied across a nonpolar liquid containing surfactant. The polarizing current measured after the application of a voltage step $0 \rightarrow V_{\mathrm{A}}$ is used to determine the conductivity $\sigma$, the concentration $n_{ \pm}$, and the mobility $\mu_{ \pm}$of charged inverse micelles, assuming $z= \pm 1$ and equal amounts of positively and negatively charged micelles.

Applying a reverse step voltage $\left(V_{\mathrm{A}} \rightarrow-V_{\mathrm{A}}\right)$ allows us to study possible interactions between charged inverse micelles and interactions at the interfaces, such as adsorption and desorption. A comparison of the total charge transfer in polarizing and reversal currents between $t=0$ and $t=t_{\mathrm{tr}}$ (the time at which all charged species reach the electrodes) yields the fraction of the charge that has disappeared due to electrochemical reactions at the interfaces. The relaxation current $\left(-V_{\mathrm{A}} \rightarrow 0\right)$ can be used to study the diffusion of charged species. This method has previously been used for PIBS (OLOA1200) ${ }^{29}$ and AOT $^{31,32}$ surfactants in nonpolar media. For OLOA1200, the current on a shorter time scale represents the motion of initially charged inverse micelles under the influence of an electric field that can be modeled accurately with drift and diffusion. ${ }^{29}$ On a longer time scale, a nonzero steady-state current exists, which can be explained by the generation of charged inverse micelles in the bulk of the solution $^{29}$ and by surface generation. ${ }^{33}$ For AOT, the generation rate of charged AOT micelles is much larger than that of charged OLOA micelles and is sufficient to maintain the equilibrium concentration of charged inverse micelles in the bulk, even when a field is applied. For this reason, studying the properties of charged AOT inverse micelles is not straightforward. We observe that most of the charged AOT inverse micelles that have arrived near the oppositely charged electrode remain very close to the surface and behave as a surface layer capacitance. This results in an exponential decrease in the transient current with a time constant that is proportional to the bulk thickness and inversely proportional to the mass fraction of AOT. ${ }^{31}$

In this article, we demonstrate that charged inverse micelles from a variety of surfactants in dodecane can be studied using the transient current measurement method. We determine the concentrations, mobilities, and hydrodynamic sizes of five surfactants: OLOA11K, Solsperse13940, Span80, Span85, and AOT for a wide range of applied voltages, cell thicknesses, and concentrations of surfactant. On the basis of our experiments, we distinguish two classes of surfactants with different behaviors, mostly determined by their different generation rates and interaction with the interface.

\section{MATERIALS AND METHODS}

The devices used for the transient current measurements consist of two glass plates coated with indium tin oxide (ITO) electrodes, with overlapping area $S=1 \mathrm{~cm}^{2}$. Glass spacer balls mixed with ultraviolet curing glue (Norland) position the two glass plates at a distance $d$. For some measurements, the ITO electrodes have been coated with an ebeam-evaporated $\mathrm{Al}_{2} \mathrm{O}_{3}$ layer with a thickness of $50 \mathrm{~nm}$ to reduce the surface generation of charged micelles and Faradaic reactions. Devices were made with thicknesses of $d=7,17,20,25$, and $27 \mu \mathrm{m}$. The thicknesses are determined from the interference fringes in the transmission spectra that are recorded with a spectrophotometer (PerkinElmer, Lambda 35). The volume between the electrodes is filled with a mixture of high-purity (99.9\%) n-dodecane (dielectric constant $\epsilon=2$ ) (VWR) and different concentrations of surfactants
AOT (Sigma-Aldrich), OLOA11000 (Merck), Solsperse13940 (Sigma-Aldrich), Span80 (Merck), and Span85 (Merck). No measures have been taken to eliminate traces of water in the mixtures or to purify the solutions.

The currents are measured using a setup based on a Keithley 428 current amplifier. Each experiment starts from a homogeneous distribution of charges after the electrodes have been short-circuited for a sufficient amount of time. At $t=0$, a voltage $V_{\mathrm{A}}$ is applied over the electrodes, and the resulting polarizing current is measured. The measured currents can be used to obtain information about transport and generation mechanisms of charges in nonpolar media.

\section{THEORY}

The measured currents are analyzed by the following model. In thermal equilibrium, a small fraction of inverse micelles is charged by different mechanisms, the two most common being disproportionation and dissociation. ${ }^{29}$ The concentrations of positive and negative charged micelles are denoted as $n_{+}$and $n_{-}\left(m^{-3}\right)$. Assuming the valency of charged micelles $z$ to be $\pm 1^{12,30}$ and from charge conservation, we can conclude that $n_{+}$ $=n_{-}$at $t=0$. In the model, we assume that positive and negative micelles have the same size and the same mobility $\mu=$ $\mu_{+}=\mu_{-}\left(\mathrm{m}^{2} \cdot \mathrm{V}^{-1} \cdot \mathrm{s}^{-1}\right)$. We believe that this is a reasonable assumption as we do not observe two different mobilities in the measurements. Because the surface of the electrodes $\left(1 \mathrm{~cm}^{2}\right)$ is much larger than the cell thickness $(\sim \mu \mathrm{m})$, the device can be considered to be a $1 \mathrm{D}$ structure.

In the absence of an electric field for a sufficient amount of time, there is a homogeneous distribution of charged micelles and an equilibrium concentration $\bar{n}=n_{+}=n$ at $t=0$. When a voltage is applied at $t=0$, drift, diffusion, and generation terms influence the concentration of charged micelles, and the transport of inverse micelles is determined by

$$
\frac{\partial n_{ \pm}}{\partial t}=D \frac{\partial^{2} n_{ \pm}}{\partial x^{2}}-\frac{\partial}{\partial x}\left(n_{ \pm} \mu_{ \pm} E\right)+\left(\frac{\partial n_{ \pm}}{\partial t}\right)_{\text {generation }}
$$

where $D$ is the diffusion constant $\left(\mathrm{m}^{2} \cdot \mathrm{s}^{-1}\right)$ given by the Einstein relation $D=\mu K_{\mathrm{B}} T / z e$, with $K_{\mathrm{B}}$ being the Boltzmann constant and $T$ being the absolute temperature $(\mathrm{K}) . E$ is the electric field $(\mathrm{V} / \mathrm{m})$, which is $V_{\mathrm{A}} / d$ at $t=0$.

A similar equation describes the variation in the concentration of neutral micelles $n_{0}$. In this article, we will simulate the currents without considering generation or recombination; therefore, $n_{0}$ is a constant. The field distribution at time $t$ is related to the potential at the electrodes $\left(\int_{0}^{d} E \mathrm{~d} x=V_{\mathrm{A}}\right)$ and the distribution of charges described by Poisson's equation:

$$
\epsilon_{0} \frac{\partial E}{\partial x}=\rho
$$

In this article, transient currents are simulated by solving the Poisson-Nernst-Planck (PNP) equations (eqs 1 and 2) without considering the generation and recombination of charged micelles. A fixed potential is applied over the electrodes, and we assume that there is no micelle flux at the electrode interfaces as the boundary condition. The focus of this article lies on the initial part of the transient current that is due to the motion of the initially present charged species rather than to the motion of newly generated charges. Initial conditions are homogeneous concentrations of charged inverse micelles and a uniform electrical field in the bulk. The electrical field is a function of the position $x$ and time $t$ and follows from the distribution of the charges and the law of Gauss. The generation reaction rate is set to zero because we are interested 

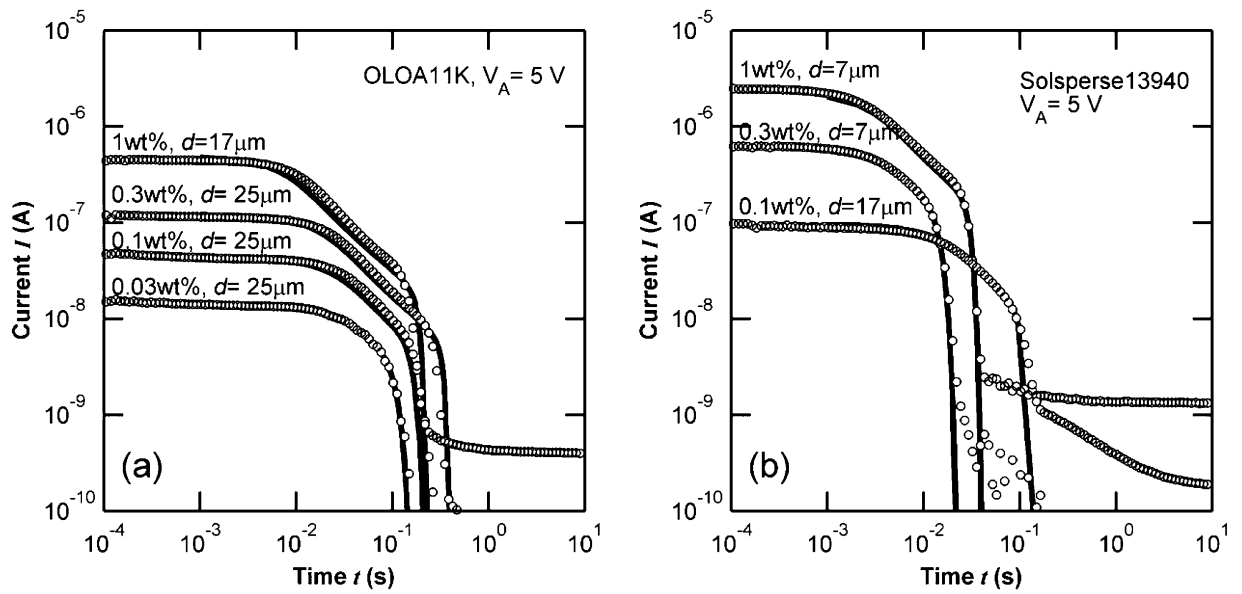

Figure 1. Transient current measurements for cells with bare ITO electrodes and 1D simulation (solid lines) for (a) OLOA11K and (b) Solsperse 13940 in dodecane systems in cells with $S=1 \mathrm{~cm}^{2}$, with a given thickness $d$ and surfactant concentration at $5 \mathrm{~V}$.

only in the current due to the initially present charged micelles. Cell parameters $V$ and $d$ are taken from the values used in the experiments. Estimated values for the concentration $n$ and the mobility $\mu$ of charged species are derived from the measurements by the following procedure. First the equilibrium concentration of charged species $\bar{n}$ is calculated by integrating the measured transient current for a voltage that is sufficiently high to lead to the complete separation of charges:

$$
n=n_{ \pm}^{t=0}=\int_{t=0}^{t_{\mathrm{tr}}} \frac{I \mathrm{~d} t}{z e S d}
$$

The integral is taken from $t=0$ to $t=t_{\mathrm{tr}}$, when the charged species have reached the electrodes. At time $t_{\mathrm{tr}}$, the current drops steeply and reaches the steady-state value. Any current after $t_{\mathrm{tr}}$ is due to the leakage and generation of new charged species and is not included in the integral. The mobility is determined from the initial current, when the field and concentrations are considered to be uniform:

$$
\mu=\frac{I_{t=0} d}{2 z e \operatorname{Sn} V_{\mathrm{A}}}
$$

The best fit for the concentration $n$ and the mobility $\mu$ is found by minimizing the average squared vertical distance between the measured and simulated transient currents on the $\log -\log$ current-time graph. Because the initial current $I_{0}$ is determined quite accurately, $n$ and $\mu$ are varied together in such a way that their product always satisfies eq 4 .

\section{RESULTS AND MODEL}

4.1. Transient Current Measurements. The polarizing current $I(t)$ is used to determine the concentration and mobility of the charged species that are initially present in the volume between the electrodes. Sets of polarizing current measurements are shown in Figures 1-3 for different surfactant concentrations of OLOA1100, Span85, Span80, AOT, and Solsperse 13940 in dodecane and for different device thicknesses. For each concentration of surfactant, the device thickness and voltage amplitude are chosen to obtain a visible decay in the polarizing current with a high value for $f$, the ratio of the initial current $I_{0}$ over the steady-state current $I_{\mathrm{ss}}$, in order to simplify the interpretation. In some cases, thinner devices have been used to reduce the flow and electrohydrodynamic effect. $^{34}$
As can be seen in Figure 1, for OLOA11K and Solsperse13940, the current drops significantly at time $t_{\mathrm{tr}}$, and $f$ is on the order of a few hundreds, at voltages higher than $1 \mathrm{~V}$ to separate the ion species. Interpreting and obtaining $n$ and $\mu$ is straightforward in these types of surfactants, as explained before.

For other surfactants such as Span80, $f$ is about 10, as illustrated in Figure 2. By properly choosing the thickness of

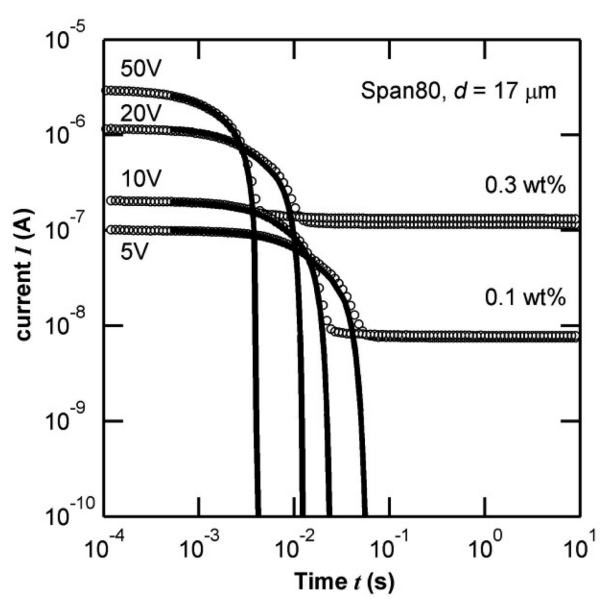

Figure 2. Transient current measurements for cells with bare ITO electrodes and 1D simulation (solid lines) for Span80 in dodecane in cells with $S=1 \mathrm{~cm}^{2}, d=17 \mu \mathrm{m}$, and 0.1 and $0.3 \mathrm{wt} \%$ for different applied voltages.

the device and the applied voltage, we can optimize the measured polarizing current and increase the factor $f$. Although the study of these types of surfactants is more critical compared to that of the first discussed group, it is still possible to observe the different stages at short and long time scales, corresponding to the initially present and newly generated inverse micelles, respectively. As can be seen in Figure 2 for Span80, at sufficiently high voltages, the measured steady-state currents become independent of the applied voltage. This phenomenon has been studied in more detail for OLOA1200. ${ }^{29}$ As expected for the case of the disproportionation of inverse micelles as the charge-generating mechanism, the saturated steady-state current is proportional to the square of the surfactant concentration (as observed in Span80, Figure 2). 

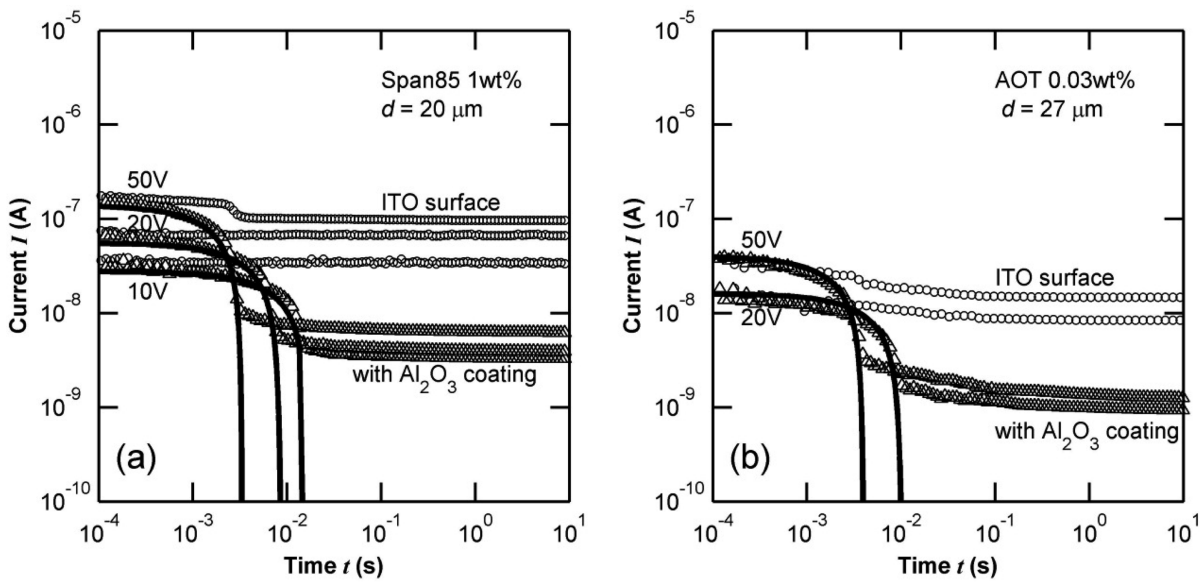

Figure 3. Transient current measurements for (a) Span85 and (b) AOT in dodecane systems for cells with bare ITO electrodes $(\mathrm{O})$ and with $\mathrm{Al}_{2} \mathrm{O}_{3}$ coating electrodes $(\triangle)$ and 1D simulation (solid lines) in cells with $S=1 \mathrm{~cm}^{2}$, with a given thickness $d$, surfactant concentration, and applied voltage.

For AOT and Span85 (Figure 3), $f$ is even lower. For these materials, the measurements with bare ITO electrodes show a ratio $f$ that is close to 1 ( $\bigcirc$, Figure 3$)$. In measurements with such a low $f$ value (with the steady-state current almost equal to the initial current), it is not possible to find the mobility and the concentration of the charged micelles.

The amplitude of the steady-state current is determined by processes such as the bulk generation of new charged inverse micelles and the surface generation of charged inverse micelles. ${ }^{33}$ Faradaic currents in the sense of molecular ions do not play a role (these should disappear with blocking insulators); therefore, only charges in the form of inverse micelles contribute to the electric current. ${ }^{31,33}$

To characterize surfactants with a high steady-state current, two approaches are used. One option to increase $f$ is to use a small cell thickness and a high voltage because this increases the initial current compared to the bulk generation current. Another option is to use devices with a thin $\mathrm{Al}_{2} \mathrm{O}_{3}$ coating on top of the ITO electrodes to reduce the surface generation current. With this method, the ratio between the initial current and the steady-state current increases to about 10, as illustrated in Figure 3, and the characterization of the initially present charged micelles becomes possible. This also indicates that an insulating layer can reduce the generation of charged inverse micelles on the surface of the cell.

4.2. Simulations. As explained in section 2, transient current measurements provide a straightforward way to obtain information about transport and generation mechanisms of charges in nonpolar media. Cell parameters $V$ and $d$ are taken from the values used in the experiments. Estimated values for the concentration $n$ and the mobility $\mu$ of charged species are derived from eqs 3 and 4 . First the equilibrium concentration of charged species $n$ is calculated by integrating the measured transient current for a voltage that is sufficiently high to lead to the complete separation of charges. Then, the mobility is determined from the initial current and the concentration.

Figures 1-3 show simulations of the transient current (solid lines) corresponding to the measurements (dots), obtained after finding the best fit for the concentration $n$ and the mobility $\mu$. Table 1 presents the best fit values of $n$ and $\mu$ and the corresponding radii $R$ of inverse micelles for each concentration of surfactants at the highest applied voltages in Figures 1-3.
Table 1. Type and Concentration of Surfactant, Measured Concentration $n$, Mobility $\mu$, and Resulting Radius $R$ of the Inverse Micelles ${ }^{a}$

\begin{tabular}{llllc}
\multicolumn{1}{c}{ surfactant } & $c($ wt $\%)$ & $n\left(\mathrm{no} . / \mathrm{m}^{3}\right)$ & $\mu\left(\mathrm{m}^{2} \cdot \mathrm{V}^{-1} \cdot \mathrm{s}^{-1}\right)$ & $R(\mathrm{~nm})$ \\
OLOA11K & 0.03 & $2.08 \times 10^{18}$ & $1.14 \times 10^{-9}$ & 5.32 \\
OLOA11K & 0.1 & $7.14 \times 10^{18}$ & $1.02 \times 10^{-9}$ & 5.94 \\
OLOA11K & 0.3 & $16.1 \times 10^{18}$ & $1.15 \times 10^{-9}$ & 5.27 \\
OLOA11K & 1 & $42.2 \times 10^{18}$ & $1.16 \times 10^{-9}$ & 5.23 \\
Solsperse 13940 & 0.1 & $13.1 \times 10^{18}$ & $0.76 \times 10^{-9}$ & 7.98 \\
Solsperse 13940 & 0.3 & $38.6 \times 10^{18}$ & $0.70 \times 10^{-9}$ & 8.66 \\
Solsperse 13940 & 1 & $150 \times 10^{18}$ & $0.70 \times 10^{-9}$ & 8.66 \\
Span80 & 0.1 & $7.18 \times 10^{18}$ & $1.50 \times 10^{-9}$ & 4.04 \\
Span80 & 0.3 & $20.8 \times 10^{18}$ & $1.48 \times 10^{-9}$ & 4.09 \\
Span85 & 1 & $0.78 \times 10^{18}$ & $2.34 \times 10^{-9}$ & 2.59 \\
AOT & 0.03 & $0.18 \times 10^{18}$ & $3.61 \times 10^{-9}$ & 1.68
\end{tabular}

${ }^{a}$ Measurements are derived from the best fit for the transient current measurements in Figures 1-3.

To calculate the size of the charged inverse micelles, we assume that all inverse micelles of these surfactants are spherical, as it has been reported in the literature. Kotlarchyk et al. reported the spherical shape of AOT in $n$-decane, ${ }^{24}$ and Guo et al. found the same results for Span 85 in hexane. ${ }^{22}$ Some works suggest that the reverse micelles formed by Span 80 are not spherical. ${ }^{35}$ However, we assume a spherical shape for Span 80 micelles, and we follow this approach in our calculations. Under this assumption, we can calculate the effective hydrodynamic radius of inverse micelles based on Stokes' law, $R=z e /(6 \pi \eta \mu)$, the results of which are summarized in Table 1 . The viscosity $\eta$ of the solutions is assumed to be equal to the viscosity of dodecane $\left(1.4 \times 10^{-3} \mathrm{~Pa} \cdot \mathrm{s}\right.$ at room temperature). The concentration of surfactants is sufficiently low $(\ll 2 \%)$ to neglect changes in the viscosity. For higher concentrations of surfactant, additional viscosity measurements are recommended.

Using the hydrodynamic radius of the inverse micelles reported in the literature ${ }^{28-31}$ and Stokes' law with the viscosity of dodecane $\eta=1.38 \times 10^{-3} \mathrm{~kg} \cdot \mathrm{m}^{-1} \cdot \mathrm{s}^{-1}$, a similar value $\mu=3.8$ $\times 10^{-9} \mathrm{~m}^{2} \cdot \mathrm{V}^{-1} \cdot \mathrm{s}^{-1}$ is obtained for the mobility of charged AOT inverse micelles. Assuming again that all the inverse micelles have the same size and monovalent charge, the concentration of charged inverse micelles using the conductive measurement 
with this estimated value of $\mu$, is $\bar{n}=n_{+}=n_{-}=8.2 \times 10^{18}\left(\mathrm{~m}^{-3}\right)$ per weight percentage of AOT.

4.3. Small versus Large Inverse Micelles. Previous studies have revealed differences between AOT and OLOA inverse micelles ${ }^{29,31}$ that deserve discussion in this context. These studies suggest that most charged AOT inverse micelles near the surface are immobilized in a Helmholtz double layer. As a result, the screening of the electric field in the bulk is mostly determined by the capacitance of the Helmholtz layer, and the current decays exponentially over a long time. ${ }^{31}$

It was observed that with high concentrations of OLOA and relatively low applied voltages, the transient current decreases considerably because the formation of a double layer with thickness $\lambda_{\mathrm{DL}}$ screens the electric field in the bulk within the socalled diffuse double layer time, $t_{\mathrm{DL}}=2 d \lambda_{\mathrm{DL}} D .^{11}$ Although other methods such as impedance spectroscopy ${ }^{36}$ or experiments with small dielectric particles ${ }^{28,37}$ can reveal the properties of a diffuse double layer for AOT, the transient currents that we measure are dominated by the large interface capacitance $C_{i}=$ $9.56 \times 10^{-2} \mathrm{~F} / \mathrm{m}^{2}$ and decrease exponentially. The time decay constant can be characterized by $C_{i} d / S \sigma$, where $\sigma$ is the conductivity of the mixture of AOT and dodecane and $S(m)$ is the surface area of the electrode. Additional measurements (not shown here) indicate that Span 85 and Span 80 behave as AOT in this respect, whereas Solsperse 13940 forms a diffuse double layer, just like OLOA11k.

The size of the inverse micelles has an influence on the level of the steady-state current as can be seen in Figures 1-3 and Table 1 . The electrostatic energy of a charged micelle with radius $R$ is $g_{\mathrm{B}}=z^{2} K_{\mathrm{B}} T \lambda_{\mathrm{B}} / 2 R,{ }^{27,28,37}$ where $\lambda_{\mathrm{B}}$ is the Bjerrum length, the distance at which two opposite charges can stay separated and their thermal energy equals the electrical energy of the ions. For univalent charges in dodecane $(\epsilon=2)$, the Bjerrum length is $28 \mathrm{~nm}$. For smaller inverse micelles, the electrostatic energy of charged micelles $g_{\mathrm{B}}$ is larger and the ratio of charged over unchanged micelles $\chi=\left(n_{+}+n_{-}\right) / n_{0}=$ $2 e^{-g_{\mathrm{B}} / K_{\mathrm{B}} T}$ is expected to be smaller. A system with smaller micelles leads to a higher reaction rate and a higher steady-state current. This matches well with Figures 2 and 3, where the levels of the steady-state currents are higher than in Figure 1.

It appears that the size of inverse micelles (Table 1) determines whether a diffuse double layer is important. Previous studies have revealed that for the small AOT inverse micelles (radius $1.6 \mathrm{~nm}$ ) the extended diffuse double layer usually does not show up and screening is due to the thin interface capacitor formed by the Helmholtz layer. ${ }^{31}$ For OLOA1200 the inverse micelles of $7 \mathrm{~nm}$ form a normal diffuse double layer. ${ }^{29}$ Additional measurements (not shown here) indicate that Span 85 and Span 80 behave as AOT in this respect, whereas Solsperse13940 forms a diffuse double layer, just like OLOA11k.

\section{DISCUSSION}

The transient current method can be used to derive the conductivity, the concentration, and the mobility of charged species in nonpolar media. This method can be used to study the dynamics and charging mechanism of surfactants in nonpolar solvents. The analysis of the current is not straightforward because different mechanisms may be present. For AOT and Span85 it is not easy to separate the contribution of initially charged micelles from the contribution of generation. Apparently the size of the inverse micelles plays an important role in the shape of the transient current $I(t)$. The smaller the size, the lower the fraction of charged inverse micelles and the higher the generation rate. In this article we demonstrate for the first time that the method can also be used for surfactants that produce small inverse micelles, by choosing small device thicknesses and high voltages and, when necessary, an insulating layer on top of the ITO electrode.

Figure 3 shows that the initial transient current can be more easily observed in devices with a layer of $\mathrm{Al}_{2} \mathrm{O}_{3}$ on top of the ITO. A similar behavior is observed for devices with different thicknesses and different mass fractions for AOT and Span85. The steady-state current in devices with an insulator is limited by the bulk generation rate of charged inverse micelles and therefore saturates at higher voltages. This behavior is more noticeable in devices with lower concentrations of AOT and Span 85.

In the transient current measurement technique we assume a unique size for the inverse micelles and study only the charged inverse micelles. By combining this method with other techniques (such as DLS or SANS), a complete set of information on charged and uncharged inverse micelles can be obtained. The strength of the transient current method is that the mobility and the concentration of charged micelles can be determined simultaneously.

In this article, the simple model for charge transport has been used on the basis of drift and diffusion, neglecting generation. This simple model can explain the shape of the initial transient current and determine the concentration and mobility of charged inverse micelles with a fitting procedure. The calculated radii of charged inverse micelles from Stokes' law (presented in Table 1) are comparable to values found in the literature. Kotlarchyk et al. studied AOT in decane using the small-angle neutron scattering technique (SANA), and they found that inverse micelles are spherical with a hydrodynamic radius of $1.6 \mathrm{~nm} .{ }^{24}$ Guo et al. have used DLS to study Span 85 in hexane. They have found a diameter of $2.8 \mathrm{~nm}$ for the inverse micelles, which is in good agreement with our results. The concentration of charged AOT inverse micelles presented in Table 1 is in good agreement with the value of $10^{17} \mathrm{~m}^{-3}$ obtained from conductivity measurements and reported by $\mathrm{Hsu}$ et al. and Roberts et al. 27,28

\section{CONCLUSIONS}

We have used transient current measurements to investigate the mobility, size, and concentration of charged inverse micelles for various surfactants in nonpolar liquid dodecane. For some surfactants, the steady-state currents are low and the analysis of the transient current is straightforward (OLOA11k, Solsperse 13940). For smaller surfactants and as a result of higher steady-state currents (Span80), the accuracy of interpretation can be increased by optimizing the device thickness and the applied voltage. For the surfactants with even higher steadystate currents and smaller sizes (AOT and Span85), the contribution of bulk and surface generation is minimized by additionally covering the electrode with an insulating layer. In this way, the concentration and mobility of the inverse micelles for all of these surfactants can be determined. The hydrodynamic radii of the inverse micelles have been calculated for the surfactants on the basis of Stokes' law, and we are able to determine the radii of inverse micelles with an accuracy of less than $1 \mathrm{~nm}$. 


\section{AUTHOR INFORMATION}

\section{Notes}

The authors declare no competing financial interest.

\section{ACKNOWLEDGMENTS}

This work has been sponsored by Merck Chemicals Ltd. Technical Centre, the Center for Nano- and Biophotonics at Ghent University, and FWO-Vlaanderen.

\section{REFERENCES}

(1) Comiskey, B.; Albert, J. D.; Yoshizawa, H.; Jacobson, J. An electrophoretic ink for all-printed reflective electronic displays. Nature 1998, 394, 253-255.

(2) Chen, Y.; Au, J.; Kazlas, P.; Ritenour, A.; Gates, H.; McCreary, M. Flexible active-matrix electronic ink display. Nature 2003, 423, 136136.

(3) Bert, T.; De Smet, H.; Beunis, F.; Neyts, K. Complete electrical and optical simulation of electronic paper. Displays 2006, 27, 50-55.

(4) Murau, P.; Singer, B. Understanding and Elimination of Some Suspension Instabilities in an Electrophoretic Display. J. Appl. Phys. 1978, 49, 4820-4829.

(5) Kazlas, P. T.; McCreary, M. D. Paperlike microencapsulated electrophoretic materials and displays. MRS Bull. 2002, 27, 894-897.

(6) Kim, C. A.; Joung, M. J.; Ahn, S. D.; Kim, G. H.; Kang, S. Y.; You, I. K.; Oh, J.; Myoung, H. J.; Baek, K. H.; Suh, K. S. Microcapsules as an electronic ink to fabricate color electrophoretic displays. Synth. Met. 2005, 151, 181-185.

(7) Novotny, V.; Hopper, M. A. Optical and Electrical Characterization of Electrophoretic Displays. J. Electrochem. Soc. 1979, 126, 2211-2216.

(8) Meng, X. W.; Tang, F. Q.; Peng, B.; Ren, J. Monodisperse Hollow Tricolor Pigment Particles for Electronic Paper. Nanoscale Res. Lett. 2010, 5, 174-179.

(9) Morrison, I. D. Electrical Charges in Nonaqueous Media. Colloids Surf., A 1993, 71, 1-37.

(10) Gibson, G. A.; Luebbe, R. H. Electrically Stable Charge Directors for Liquid Toners for High-Speed Electrophotographic Printing. J. Imaging Technol. 1991, 17, 207-209.

(11) Beunis, F.; Strubbe, F.; Marescaux, M.; Beeckman, J.; Neyts, K.; Verschueren, A. R. M. Dynamics of charge transport in planar devices. Phys. Rev. E 2008, 78, 1 .

(12) Verschueren, A. R. M.; Notten, P. H. L.; Schlangen, L. J. M.; Strubbe, F.; Beunis, F.; Neyts, K. Screening and Separation of Charges in Microscale Devices: Complete Planar Solution of the PoissonBoltzmann Equation. J. Phys. Chem. B 2008, 112, 13038-13050.

(13) Strubbe, F.; Beunis, F.; Marescaux, M.; Verboven, B.; Neyts, K. Electrokinetics of colloidal particles in nonpolar media containing charged inverse micelles. Appl. Phys. Lett. 2008, 93, 254106.

(14) Kim, J. Y.; Garoff, S.; Anderson, J. L.; Schlangen, L. J. M. Movement of colloidal particles in two-dimensional electric fields. Langmuir 2005, 21, 10941-10947.

(15) Zoeller, N.; Blankschtein, D. Experimental determination of micelle shape and size in aqueous solutions of dodecyl ethoxy sulfates. Langmuir 1998, 14, 7155-7165.

(16) Gracia, C. A.; Gomez-Barreiro, S.; Gonzalez-Perez, A.; Nimo, J.; Rodriguez, J. R. Static and dynamic light-scattering studies on micellar solutions of alkyldimethylbenzylammonium chlorides. J. Colloid Interface Sci. 2004, 276, 408-413.

(17) Kinugasa, T.; Kondo, A.; Nishimura, S.; Miyauchi, Y.; Nishii, Y.; Watanabe, K.; Takeuchi, H. Estimation for size of reverse micelles formed by AOT and SDEHP based on viscosity measurement. Colloids Surf., A 2002, 204, 193-199.

(18) Lemyre, J. L.; Lamarre, S.; Beaupre, A.; Ritcey, A. M. A New Approach for the Characterization of Reverse Micellar Systems by Dynamic Light Scattering. Langmuir 2010, 26, 10524-10531.

(19) Berne, B. J.; Pecora, R. Dynamic Light Scattering: With Applications to Chemistry, Biology, and Physics; Wiley: New York, 1976.
(20) Schmitz, K. S. An Introduction to Dynamic Light Scattering by Macromolecules; Academic Press: Boston, 1990.

(21) Evans, D. F.; Wennerström, H. K. The Colloidal Domain: Where Physics, Chemistry, Biology, and Technology Meet. VCH Publishers: New York, 1994.

(22) Guo, Q.; Singh, V.; Behrens, S. H. Electric Charging in Nonpolar Liquids Because of Nonionizable Surfactants. Langmuir 2010, 26, 3203-3207.

(23) Eicke, H. F. Aggregation in Surfactant Solutions - Formation and Properties of Micelles and Micro-Emulsions. Pure Appl. Chem. 1980, 52, 1349-1357.

(24) Kotlarchyk, M.; Huang, J. S.; Chen, S. H. Structure of AotReversed Micelles Determined by Small-Angle Neutron-Scattering. J. Phys. Chem. 1985, 89, 4382-4386.

(25) Poovarodom, S.; Berg, J. C. Effect of particle and surfactant acidbase properties on charging of colloids in apolar media. J. Colloid Interface Sci. 2010, 346, 370-377.

(26) Gacek, M.; Bergsman, D.; Michor, E.; Berg, J. C. Effects of Trace Water on Charging of Silica Particles Dispersed in a Nonpolar Medium. Langmuir 2012, 28, 11633-11638.

(27) Hsu, M. F.; Dufresne, E. R.; Weitz, D. A. Charge stabilization in nonpolar solvents. Langmuir 2005, 21, 4881-4887.

(28) Roberts, G. S.; Sanchez, R.; Kemp, R.; Wood, T.; Bartlett, P. Electrostatic charging of nonpolar colloids by reverse micelles. Langmuir 2008, 24, 6530-6541.

(29) Strubbe, F.; Verschueren, A. R. M.; Schlangen, L. J. M.; Beunis, F.; Neyts, K. Generation current of charged micelles in nonaqueous liquids: Measurements and simulations. J. Colloid Interface Sci. 2006, 300, 396-403.

(30) Beunis, F.; Strubbe, F.; Karvar, M.; Drobchak, O.; Brans, T.; Neyts, K. Inverse micelles as charge carriers in nonpolar liquids: Characterization with current measurements. Curr. Opin Colloid Interface Sci. 2013, 18, 129-136.

(31) Karvar, M.; Strubbe, F.; Beunis, F.; Kemp, R.; Smith, A.; Goulding, M.; Neyts, K. Transport of Charged Aerosol OT Inverse Micelles in Nonpolar Liquids. Langmuir 2011, 27, 10386-10391.

(32) Novotny, V. Electrical-Conduction in Surfactant-Water-Nonaqueous Liquid-Systems. J. Electrochem. Soc. 1986, 133, 1629-1636.

(33) Beunis, F.; Strubbe, F.; Marescaux, M.; Neyts, K.; Verschueren, A. R. M. Micellization and adsorption of surfactant in a nonpolar liquid in micrometer scale geometries. Appl. Phys. Lett. 2010, 97, 18.

(34) Strubbe, F.; Vanbrabant, P. J. M.; Beunis, F.; Verboven, B.; Karvar, M.; Neyts, K. In-plane electrophoresis in nonpolar liquids: Measurements and simulations. Colloids Surf., A 2011, 376, 89-96.

(35) Abou-Nemeh, I.; Bart, H. J. Microstructures in the system water/D2EHPA/span-80/n-dodecane. Langmuir 1998, 14, 44514459.

(36) Barbero, G. Influence of adsorption phenomenon on the impedance spectroscopy of a cell of liquid. Phys. Rev. E 2005, 71, 062201.

(37) Sainis, S. K.; Merrill, J. W.; Dufresne, E. R. Electrostatic Interactions of Colloidal Particles at Vanishing Ionic Strength. Langmuir 2008, 24, 13334-13347. 